\title{
Secure Login System Using QR-Image
}

\author{
Shreya Dutta \\ Department of Computer \\ Engineering \\ D.Y. Patil Institute of \\ Engineering and Technology \\ Pune, India
}

\author{
Shalu Singh \\ Department of Computer \\ Engineering \\ D.Y. Patil Institute of \\ Engineering and Technology \\ Pune, India
}

\author{
Pratiksha Ghodke \\ Department of Computer \\ Engineering \\ D.Y. Patil Institute of \\ Engineering and Technology \\ Pune, India
}

\begin{abstract}
In this paper, we are introducing a new idea to secure the login by generating a QR code (Quick Response Code) image. QR code is an extension of a barcode. Since QR code has a great storage capacity than a barcode, it is used to carry or store different messages. In today's era, security is a very crucial issue to handle. So this is the aim of this paper. It mainly focuses on ciphering the generated unique user-id to a secure QR code which will then be converted to a QR-image. Later this image will be segmented by using Image Segmentation. This will not only eliminate the traditional password-based system but will also reduce the chance of attack.
\end{abstract}

Keywords: QR code; Cryptography; Segmentation; Cipher; Security.

\section{INTRODUCTION}

In this world full of technologies, security is becoming an important thing to take care of. These days due to weak security systems, there are many phishing attacks and frauds have taken place in the present digital world. These attackers can be in any form be it a hacker or an intruder who are always ready to misuse every person's personal data. So every individual's data needs to be kept secure. Keeping an individual's personal valuable data like passport information, bank statements, social security number etc is very important and it is also not possible for every individual to carry their documents with them everywhere. Even if they try to save their documents in any of the available online storage applications like Google drive, I cloud etc., there's a high possibility that the data may be lost or hacked.

For this purpose, the field of cryptography (data-hiding algorithm) and image segmentation is used in this study. Cryptography is the study of techniques for safe communication in the presence of third parties. So the existing problem can be solved by encrypting the data and hiding it in the form of QR code which can be kept with the user at any time. Image segmentation is the process of partitioning a digital image into multiple segments. The goal of segmentation is to simplify and/or change the representation of an image into something that is more meaningful and easier to analyze.

\section{QR CODE}

QR code abbreviated from Quick Response Code is the trademark for a type of two-dimensional barcode, which was first designed in 1994 for the automotive industry in Japan. Since barcode has many drawbacks it is not of much use. A QR code uses four standardizing modes: numeric, alphanumeric, byte/binary and kanji. It stores data efficiently and extensions may also be used.
The QR code is very popular nowadays because of its fast readability and greater storage capacity than standard UPC barcode. There are many applications of QR code. Some of them are product tracking, item identification, time tracking, document management, and general marketing.

A QR code includes of black modules that are arranged in a square grid on a white background, which can be interpreted by mobile applications like camera/scanners and processed using Reed Solomon error correction. The required data are then extracted from patterns that are present in both horizontal and vertical components of the QR image.

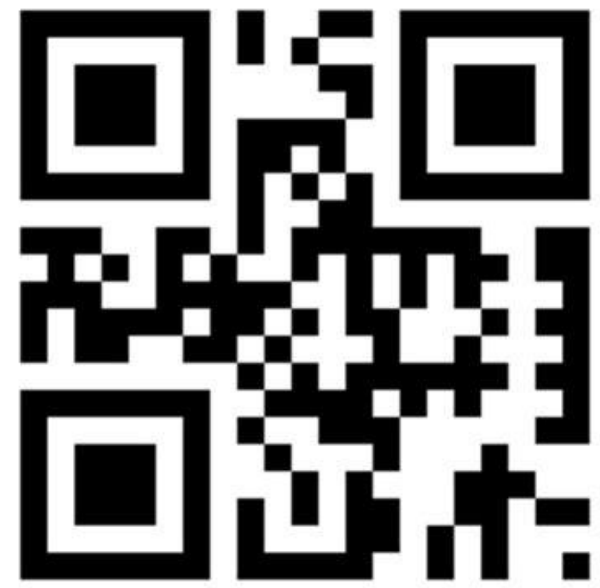

Figure. 1 QR code 


\section{PROPOSED SYSTEM}

In the paper, we are using data hiding algorithm and image segmentation for any login system. In this proposed system, there will be two modules: admin and user. The admin can see how many users are there and also he/she can delete the users. The user can register himself/herself in the system and a unique QR code will be generated for that particular user in $\mathrm{QR}$ image format. He/she can auto-download the QR image in his/her respective systems and next time when they will login, they only have to upload that particular image instead of their username and password. The QR image will be divided into two parts with the help of image segmentation. One part of the QR image will be at the server and the other part will be available to the user in the QR image format. They can also save this image in their mobile devices and this will also enable them to login on their devices. While logging, the user will upload this image that is available on his/her system /device and this image will be matched with the image present on the server. Then the server will validate it. And when the validation will be successful, the user can successfully logged-in on to that system.

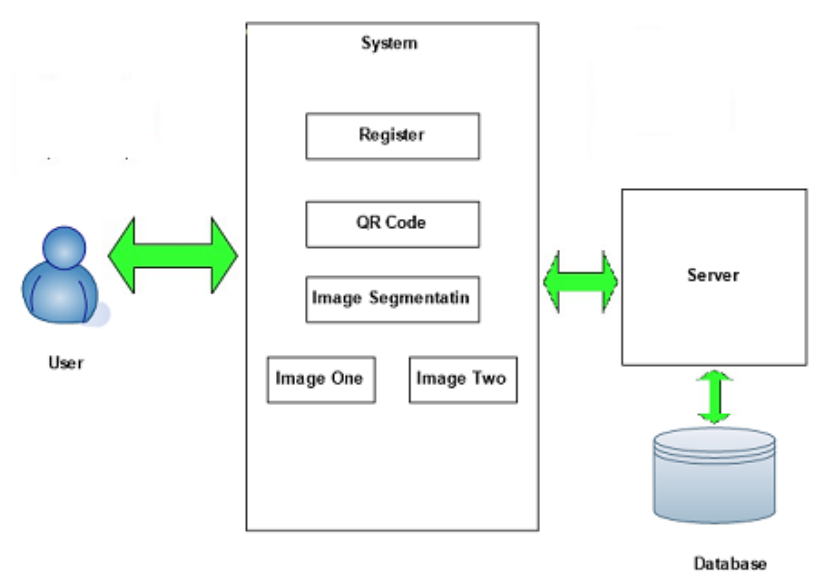

Figure. 2 System Architechture

\section{ALGORITHM}

\subsection{Algorithm Of convert plaintext to $Q R$ code}

Step 1: write message (text).

Step 2: generate QR code for the message.

Step 3: save $\mathrm{QR}$ image as $\mathrm{P}$.

\subsection{Algorithm of Convert Key to $Q R$ code} Step 1: write key as numbers or text.

Step 2: generate $\mathrm{QR}$ code for the key.

Step 3: save QR image as K.

\subsection{Algorithm of get Begin Indies of Data Area in QR}

Step 1: start.

Step 2: Do loop to get beginning of data area in plain.bmp with width $\mathrm{i}$ as height $\mathrm{j}$.

Step 3: end.

\subsection{Algorithm of Encryption}

Step 1: start.

Step 2: load QR image P.

Step 3: load QR image k.

Step 4: define cipher as bitmap file with dimensions width (wd) \& height (hg).

Step 5:Call function to put $\mathrm{P}(0)(0)$ to $\mathrm{P}(\mathrm{i})(\mathrm{j})$ in cipher $(0)(0)$ to cipher(i)(j).

Step 6: loop statement $x=i$ loop statement $y=j$ $\operatorname{cipher}(\mathrm{x})(\mathrm{y})=\mathrm{P}(\mathrm{x})(\mathrm{y})$ XOR $\mathrm{k}(\mathrm{x})(\mathrm{y})$ next $\mathrm{y}, \mathrm{x}$.

Step7: end.

\subsection{Algorithm of Putting Key in Cipher Bitmap File}

Step 1: start.

Step 2: binarization each character or number in key as 8bit. Step 3: loop statement $1<$ key (length) If $(\operatorname{key}(1)=255)$ $\operatorname{Key}(1)=254$; Else $\operatorname{Key}(1)=1$; End if.

Step 4: if statement If $(\operatorname{key}(1)=255) \quad \operatorname{Key}(1)=253$; Else $\operatorname{Key}(1)=2$; End if.

Step 5: end.

\subsection{Algorithm of Getting Key from Cipher Bitmap File}

Step 1: start.

Step 2: loop statement until key(l) $=253$ or 2

If $(\operatorname{key}(1)=254$ or 1$)$

Str=concat(str,'1');

Else $\quad$ Str=concat(str,'0');

End if.

Step 3: collect each 8bit in str and get character of this collection.

Step 4: end.

\subsection{Algorithm of Decryption}

Step 1: start.

Step 2: load QR image c.

Step 3: get width (wd) \& height (hg) of c.

Step 4: define plain as bitmap file with dimensions width (wd) $\&$ height (hg).

Step 5:Call function to put $\operatorname{cipher}(0)(0)$ to $\operatorname{cipher(i)(j)}$ in plain(0)(0) to plain(i)(j).

Step 6: loop statement $\mathrm{x}=\mathrm{i}$

loop statement $\mathrm{y}=\mathrm{j}$ 
$\operatorname{plain}(\mathrm{x})(\mathrm{y})=\operatorname{cipher}(\mathrm{x})(\mathrm{y}) \operatorname{XOR} \operatorname{key}(\mathrm{x})(\mathrm{y})$

next $y, x$.

Step 7: end.

\subsection{Algorithm of Image Segmentation}

Step1:Define the neighborhood of each feature (random variable in MRF terms). Generally this includes 1st order or 2nd order neighbors.

Step2: Set initial probabilities $P\left(f_{i}\right)>$ for each feature as 0 or

Step3: where $f_{i} \in \Sigma$ is the set containing features extracted for pixel $i$ and define an initial set of clusters.

Step4:Using the training data compute the mean $\left(\mu_{i}\right)$ and variance $\left(\sigma_{l i}\right)$ for each label. This is termed as class statistics.

Step5:Compute the marginal distribution for the given labeling scheme $P\left(f_{i} \mid l_{i}\right)$ using Bayes' theorem and the class statistics calculated earlier. A Gaussian model is used for the marginal distribution.

Step 6: Calculate the probability of each class label given the neighborhood defined previously.

Clique potentials are used to model the social impact in labeling.

Step 7: Iterate over new prior probabilities and redefine clusters such that these probabilities are maximized. This is done using a variety of optimization algorithms described below.

Step 8: Stop when probability is maximized and labeling scheme does not change.

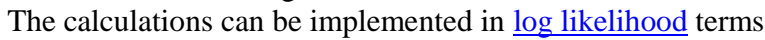
as well.

\section{CONCLUSION}

Secure login system using QR code can be used in large scope for solving any real time problems of memorizing different user-id and password. This will reduce the headache of getting confused with different user-id and password for different websites.

\section{ACKNOWLEDGMENTS}

We would like to express our special thanks of gratitude to our guide Prof. Sujay Pawar, our project coordinator head Prof. Deepti Deshmukh as well as our head of the department Prof. Mangesh Manake who gave us the golden opportunity to do this project on the topic Secure Login System Using QRimage, which also helped us in doing a lot of Research and we came to know about so many new things. We are really thankful to them.

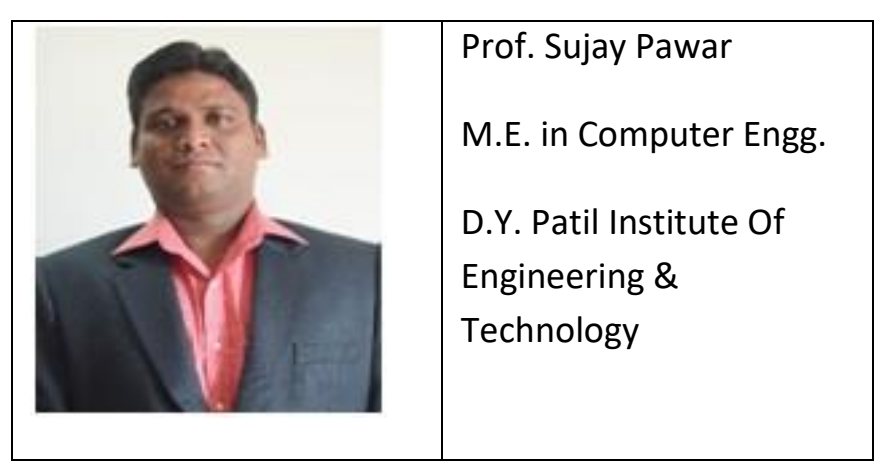

\section{REFERENCES}

[1] Basheer N. Ameen, Sawsan K. Thamer, "A Novel Method for Ciphering a Message Based on QR Codes" in International Journal of Scientific Engineering Research, Volume 8, Issue 4, 2017.

[2] P. Kieseberg, M. Mulazzani, L. Munroe, S. Schrittwieser, M. Sinha, et al., "QR code security" in Proceedings of the 8th International conference on Advances in Mobile Comuting and Multimedia, 2010.

[3] S.Balaji, Dr.N.Pughazendi, S.E.Praveenkumar, V.Vishal, R.Vignesh, "E-Medical Application using QR code with OTP generation " in International Journal of Innovative Research in Science, Engineering and Technology, 2018.

[4] S. Dey, S. Agarwal and A. Nath "Confidential Encrypted Data Hiding and Retrival Using OR Authentication System " in International Conference on, 2013. 